\title{
openheart Coronary artery disease is associated with persistent lower quality of life in women
}

To cite: Gencer B, Girardin F. Coronary artery disease is associated with persistent lower quality of life in women. Open Heart 2015:2:e000305. doi:10.1136/openhrt-2015000305

Accepted 30 June 2015

\section{Linked}

http://dx.doi.org/10.1136/ openhrt-2014-000231

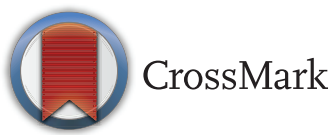

${ }^{1}$ Cardiology Division, University Hospital of Geneva, Geneva, Switzerland ${ }^{2}$ Medical Directorate, University Hospital of Geneva, Geneva, Switzerland ${ }^{3}$ Department of Anesthesiology, Intensive Care and Clinical Pharmacology and Toxicology, University Hospitals and University of Geneva, Faculty of Medicine, Geneva, Switzerland

Correspondence to Dr Baris Gencer; Baris.Gencer@hcuge.ch
Gijsberts et al reported the unfavourable impact of coronary artery disease (CAD) on the health-reported quality of life (HRQoL) in women using data from the Utrecht Coronary Biobank cohort. (Add Ref openhrt-2014-0000231.R2) The mean selfrated health status based on the EuroQol-5D (EQ-5D) was significantly lower in women compared with men $(6.46 \pm 1.40$ vs $6.84 \pm 1.49$, $\mathrm{p}<0.001)$. In all the domains of the RAND-36, women reported poorer outcomes than men, especially for physical and social functions. In addition, compared to the general Dutch population, women with CAD had significantly poorer HRQoL compared with men. This study adds relevant clinical implications of the secondary prevention of CAD in patients within a large sample (1020 men and 401 women with $\mathrm{CAD}$ ) and gives an accurate description of the impaired domains of the HRQoL in patients with CAD. The persisting poorer outcomes of HRQoL in women suggest that genderrelated factor remains a major issue regardless of the disease severity and treatment.

Recently, data from among patients who had premature acute coronary syndromes (ACS) taken from the GENESIS-PRAXY study (GENdEr and Sex determInantS of cardiovascular disease: from bench to beyondPremature Acute Coronary SYndrome) suggest that women had poorer HRQoL (measured by the Short Form 12 (SF-12) and Seattle Angina Questionnaire (SAQ)) than men 1 year after ACS. ${ }^{1}$ Gender-related factors, such as femininity score, social support and housework responsibility, are important predictors of HRQoL, and appear to be more important than sex and medical conditions. These recent data underlie the tremendous effect of premature ACS on productivity and opportunity costs in younger patients, especially in women. ${ }^{1}$ Indeed, public health interventions, such as systematic cardiac rehabilitation, should be implemented to improve perceived health status in the CAD population, especially in women.

As HRQoL includes multidimensional dimensions, such as patients' illness perception, instead of intrinsic disease outcomes (eg, cardiovascular morbidity or mortality), it is important to focus attention on these outcomes in patients with CAD. ${ }^{23}$ Several instruments are valid to measure HRQoL and these can be classified into two groups: generic and disease-specific instruments (box 1). Selfreported health status by visual analogue scale (VAS) has been used in large cardiovascular studies, such as the Bypass Angioplasty Revascularisation Investigation 2 Diabetes (BARI 2D) ${ }^{4}$ Self-rated health status measurement has been associated with clinical objective outcomes, but the variance of estimates was influenced by subjective perception. ${ }^{5}$ This might be particularly important in younger patients with disease who tend to evaluate their health more negatively. ${ }^{5}$ In addition, subjective evaluation of the health status in patients with CAD could be additionally influenced by socioeconomic and psychological factors, based on elicited preferences using the EQ-5D instrument. ${ }^{6}$

Health economic studies need input parameters to capture health benefits and utility measured by means of quality-adjusted life years (QALY) as standardised metrics. The health utility is expressed on a scale between 0 (death) and 1 (perfect health), and is derived from generic instruments such as VAS or EQ-5D. ${ }^{7}$ Recent cost-effectiveness analyses and health economic evaluation did not routinely derive health utility estimates according to gender. ${ }^{8}{ }^{9}$ However, data from observational studies, as the one published by Gijsberts $e t$ al, suggest that aggregated utility estimates are not accurate, which emphasises the need for more specific scenario analyses.

In conclusion, integrating HRQoL in medical decisions or treatment outcomes is increasingly recommended by the European 
Box 1 Instruments used for the measurement of health-related quality of life in patients with CAD

\section{Generic instruments}

- Visual analogue scale (VAS). Vertical scale from 0 (worst imaginable) to 100 (best imaginable).

- EuroQol-5D (EQ-5D). Mobility, self-care, usual activities, pain/ discomfort and anxiety/depression.

-12-Item Short Form Health Survey (SF-12). General health, physical functioning, role-physical, bodily pain, vitality, social functioning, role-emotional and mental health.

- Research and Development 36-item (RAND-36)/36-Item Short Form Health Survey (SF-36). Physical functioning, mental functioning, social functioning, physical role limitations, emotional role limitations, pain, vitality, general health and health change.

- Sickness Impact Profile (SIP). Mobility, ambulation, domestic affairs, social interaction, behaviour, communication, recreation, eating, work, sleep, emotions and self-care.

Disease-specific instruments

- Seattle Angina Questionnaire (SAQ). Physical limitations, angina stability, angina frequency, treatment satisfaction and disease perception/quality of life.

- Quality of Life after Myocardial Infarction (QLMI/MacNew) questionnaire. Physical limitations, emotional and social functioning.

- Angina Pectoris Quality of Life Questionnaire (APQLQ). Physical activity, somatic symptoms, emotional distress, and life satisfaction.

- Myocardial Infarction Dimensional Assessment Scale (MIDAS). Physical activity, insecurity, emotional reaction, dependency, diet, concerns over medications and side effects.

- Cardiovascular Limitations and Symptoms Profile (CLASP). Angina, shortness of breath, ankle swelling and tiredness.

and American guidelines for ACS management. HRQoL is as an essential outcome in patients with CAD for cardiologists and policymakers for future healthcare interventions: the evaluation of novel technologies by health authorities needs contemporary reference values to assess appropriately the resource use and the health utility gain from these given the background of increased budget constraints. ${ }^{1011}$

Competing interests None declared.

Provenance and peer review Commissioned; internally peer reviewed.

\section{REFERENCES}

1. Leung Yinko SS, Pelletier R, Behlouli $\mathrm{H}$, et al. Health-related quality of life in premature acute coronary syndrome: does patient sex or gender really matter? J Am Heart Assoc 2014;3. doi:10.1161/JAHA. 114.000901

2. Nikolic $\mathrm{E}$, Janzon $\mathrm{M}$, Hauch $\mathrm{O}$, et al. Cost-effectiveness of treating acute coronary syndrome patients with ticagrelor for 12 months: results from the PLATO study. Eur Heart J 2013;34:220-8.

3. Schweikert B, Hunger M, Meisinger C, et al. Quality of life several years after myocardial infarction: comparing the MONICA/KORA registry to the general population. Eur Heart $J$ 2009;30:436-43.

4. Thomas SB, Sansing VV, Davis A, et al. Racial differences in the association between self-rated health status and objective clinica measures among participants in the BARI 2D trial. Am J Public Health 2010;100(Suppl 1):S269-76.

5. Kaplan G, Baron-Epel O. What lies behind the subjective evaluation of health status? Soc Sci Med 2003;56:1669-76.

6. Xie J, Wu EQ, Zheng ZJ, et al. Patient-reported health status in coronary heart disease in the United States: age, sex, racial, and ethnic differences. Circulation 2008;118:491-7.

7. Perneger TV, Combescure C, Courvoisier DS. General population reference values for the French version of the EuroQol EQ-5D health utility instrument. Value Health 2010;13:631-5.

8. Janzon M, James S, Cannon CP, et al. Health economic analysis of ticagrelor in patients with acute coronary syndromes intended for non-invasive therapy. Heart 2015;101:119-25.

9. Cowper PA, Pan W, Anstrom KJ, et al. Economic analysis of ticagrelor therapy from a U.S. perspective: results from the PLATO study. J Am Coll Cardiol 2015;65:465-76.

10. Hamm CW, Bassand JP, Agewall S, et al. ESC Guidelines for the management of acute coronary syndromes in patients presenting without persistent ST-segment elevation: The Task Force for the management of acute coronary syndromes (ACS) in patients presenting without persistent ST-segment elevation of the European Society of Cardiology (ESC). Eur Heart $J$ 2011;32:2999-3054.

11. Amsterdam EA, Wenger NK, Brindis RG, Jr, et al. 2014 AHA/ACC guideline for the management of patients with non-ST-elevation acute coronary syndromes: a report of the American College of Cardiology/American Heart Association Task Force on Practice Guidelines. Circulation 2014;130:e344-426. 